\title{
SEMI-AUTOMATED SETUP OF CFD SIMULATIONS OF RECTANGULAR CYLINDERS AND STREAMLINED BOX DECKS
}

\author{
FÉLIX NIETO, ANTONIO J. ÁLVAREZ, SANTIAGO HERNÁNDEZ \& ANTÍA TROCHE \\ School of Civil Engineering, University of La Corunna, Spain
}

\begin{abstract}
A piece of software that acts as a Graphical User Interface (GUI) for the setup of computational fluid dynamics (CFD) models that are solved by means of the open source code OpenFOAM, is presented. The software is extensively described, with emphasis in the generation of block structured meshes using hexahedral elements. This computer program has been developed aiming at being applied in wind engineering problems of interest in civil engineering, such as the computation of force coefficients, flutter derivatives and vortex-induced vibrations. It has been devised to deal efficiently with rectangular cylinders and streamlined box decks. This software demands limited intervention from the user, and its core routines can be embedded in automated design processes such as parametric or optimal design problems in wind engineering. Two application examples have been considered: static and forced oscillation simulations of both, a side ratio 2:1 rectangular cylinder and a streamlined box deck. It has been found that this software is an efficient tool for the setup of URANS simulations in OpenFOAM, while the numerical results obtained for the studied aerodynamic and aeroelastic phenomena show good agreement with wind tunnel data, and their level of accuracy is equivalent to other CFD-based simulations.

Keywords: CFD, GUI, flutter derivatives, force coefficients, OpenFOAM, rectangular cylinder, streamlined box deck, vortex induced vibration.
\end{abstract}

\section{INTRODUCTION}

The interest in fluid-structure interaction (FSI) problems has steadily grown in the last decades. There are many industrial problems of this kind, such as vortex-induced vibration of buildings, self-induced excitation of bridge decks, vibrations of wind turbine blades or rainwind-induced vibrations of cables, which require an accurate modeling and in depth analysis in order to anticipate the structure's behavior. Besides, the dramatic advances in the field of computational simulation of coupled fluid-structure problems have allowed a better understanding of the involved phenomena and have decreased the analysis burden.

Moreover, the available codes for numerically simulate FSI problems have extended their capabilities and the advances in hardware resources have allowed researchers and engineers to face problems whose computational demands could have not been met a few years earlier. Also, the development of general purpose open source CFD software packages has made this numerical approach available for a wider sector of the engineering community. This is remarkable in the frame of its intensive application in massive cluster based computations.

Amongst the open source CFD codes, perhaps the most widely used is OpenFOAM. The setup process in this piece of software is based on the generation of text files (dictionaries) which are allocated according to a rigid directory scheme. The aforementioned files contain data such as the geometry of flow domain, the finite volume mesh definition, the undisturbed flow characteristics, turbulence modeling data, numerical settings for the solver or forced oscillation parameters for problems comprising dynamic meshes. The main drawback of this text files based approach is the existence of a learning curve for new users which has a steeper slope than other graphical user interface (GUI) based CFD packages. On the other 
hand, this approach is particularly suitable for developing pieces of software which introduce modifications in the setup text files, producing modified cases from a reference one. Afterwards, all the cases can be simultaneously solved using a cluster of computers, speeding up significantly both, the definition of the complete set of cases to be analyzed, and the problem solving process.

Furthermore, this capability can be extended to design oriented problems such as parametric design problems, where a certain parameter, or set of parameters, (the geometric dimensions of the cross section of a bluff body for instance), can be systematically modified, and the correspondent CFD cases recursively generated, with the aim of assessing its effect in the body's aerodynamic or aeroelastic response of interest for the designer [1].

Taking a further step, the aforementioned capability can be applied in optimum design problems considering aerodynamic and aeroelastic constraints. Along a generic numerical optimization problem, the design variables are modified by the mathematical algorithm in order to minimize (or maximize) the objective function while satisfying the prescribed set of design and behavior constraints. In this case, the optimization engine can call this code and provide the values of the modified design variables (for instance the deck width and depth in a bridge design problem) with the purpose of generating the analysis cases required to perform the next iteration in the numerical optimization process. It is remarkable that, since the finite volume grid can be parametrically defined by means of the software, geometric design variables could be tackled in a shape optimization problem, which is the main challenge in the aeroelastic optimum design of bridge decks [2].

In this work a bespoke piece of software has been developed using the MATLAB language for creating a GUI that allows defining the setup of aerodynamic and FSI problems for rectangular cylinders (rectangular prisms) and box girders of bridges using the CFD package OpenFOAM. In this manner, the finite volume mesh can be parametrically defined from the user's input in the GUI, along with the main data for setting-up the simulation. The code generates the folders and files required for defining the OpenFOAM case, which can be solved afterwards. To the authors' knowledge, there is not available software that can simultaneously complete these tasks for civil engineering applications.

Two are the types of geometries considered by this piece of software: rectangular cylinders and streamlined closed box cross sections. Rectangular cylinders are of interest since they are simple geometries which can be considered as basic bluff bodies which can suffer aeroelastic phenomena of interest in bridge engineering such as vortex induced vibration or flutter. At the same time, rectangular cylinders are often present in the built environment and they are of interest in industrial applications, such as the vortex induced vibrations suffered, for instance, by the Alconetar arch bridge during construction [3]. Closed box cross sections are a common choice for decks in long span bridges such as the Great Belt or the Tsing Ma bridges. Computing the force coefficients or the flutter derivatives of this type of section is of utmost interest for determining the critical flutter speed and the buffeting response. In fact, the numerical computation of the aerodynamic and aeroelastic behavior of rectangular cylinders and box decks is an active field of research nowadays (see Patruno, 2015 [4] or Haque et al. 2015 [5], amongst many others).

In this paper, sections 2 and 3 are devoted to the general formulation of the governing equations for flow modelling and the wind phenomena of interest. Then, in section 4, the FLUSINI (FLUid Structure INteraction Interface) software is described, paying special attention to the way the GUI is organized and the required sets of data that must be supplied by the user in each form. In the next section, the results obtained for two application cases (2:1 rectangular cylinder and a streamlined box deck) are presented. Emphasis has been put 
on the comparison of the obtained results for the static and forced to oscillate bodies with experimental and numerical data reported in the literature. The main conclusions that can be drawn from this work are summarized at the end of the document.

\section{GOVERNING EQUATIONS}

The Reynolds-Averaged Navier Stokes (RANS) equations assuming incompressible flow are used for modelling the flow past a bluff body. The conservation of mass and momentum are [6]:

$$
\begin{gathered}
\frac{\partial U_{i}}{\partial x_{i}}=0 \\
\rho \frac{\partial U_{i}}{\partial t}+\rho U_{j} \frac{\partial U_{i}}{\partial x_{j}}=-\frac{\partial P}{\partial x_{i}}+\frac{\partial}{\partial x_{i}}\left(2 \mu S_{i j}-\rho \overline{u_{i}^{\prime} u_{j}^{\prime}}\right),
\end{gathered}
$$

where $U_{i}$ is the mean velocity vector, $x_{i}$ is the position vector, $t$ is time, $\rho$ is the density of the fluid, $u$ ' is the fluctuating velocity, representing the overbar the time average, $P$ is mean pressure, $\mu$ is viscosity and $S_{i j}$ is the mean strain-rate tensor. The specific Reynolds stress tensor is defined as:

$$
\tau=-\overline{u_{i}^{\prime} u_{j}^{\prime}}
$$

which must be modeled using the Boussinesq assumption:

$$
\tau_{i j}=2 v_{T} S_{i j}-\frac{2}{3} k \delta_{i j}
$$

where $V_{T}$ is the kinematic eddy viscosity, and $k$ is the kinetic energy per unit mass of the turbulent fluctuation.

In the application cases reported in this work Menter's $k-\omega$ SST turbulence model for incompressible flow is adopted [7]. Further details, particularly on the Arbitrary Lagrangian Eulerian (ALE) formulation adopted for moving bodies, may be found in [8].

\section{WIND ENGINEERING PHENOMENA FORMULATION}

\subsection{Force coefficients definition}

Force coefficients are defined as follows:

$$
C_{D}=\frac{\bar{D}}{\frac{1}{2} \rho U^{2} B}, C_{L}=\frac{\bar{L}}{\frac{1}{2} \rho U^{2} B}, C_{M}=\frac{\bar{M}}{\frac{1}{2} \rho U^{2} B^{2}}
$$

In eqn. (7), $\bar{D}$ is the mean drag force per span length, $\bar{L}$ is the mean lift force per span length and $\bar{M}$ is the mean twist moment per unit of span length, positive clockwise. $U$ is the reference flow velocity, $\rho$ is the air density and $B$ is a reference dimension, the rectangular cylinder or the deck widths.

3.2 Computation of flutter derivatives from forced oscillations in pitch and heave degrees of freedom

Flutter derivatives are non-analytical parameters which relate the self-excited aerodynamic forces with the velocities and movements of the structure. They are of interest in the study 
of the flutter phenomenon. These parameters are usually identified from wind tunnel tests, but more recently, CFD based simulations have been applied.

According to Simiu and Scanlan [9], the aeroelastic forces on a bridge deck, considering two degrees of freedom (heave and pitch) can be written as follows:

$$
\begin{aligned}
& L_{a e}=\frac{1}{2} \rho U^{2} B\left[K H_{1}^{*} \frac{\dot{h}}{U}+K H_{2}^{*} \frac{B \dot{a}}{U}+K^{2} H_{3}^{*} a+K^{2} H_{4}^{*} \frac{h}{B}\right] \\
& M_{a e}=\frac{1}{2} \rho U^{2} B^{2}\left[K A_{1}^{*} \frac{\dot{h}}{U}+K A_{2}^{*} \frac{B \dot{\alpha}}{U}+K^{2} A_{3}^{*} a+K^{2} A_{4}^{*} \frac{h}{B}\right] .
\end{aligned}
$$

In the former equations, $L_{a e}$ is the aeroelastic force per unit of span length, $M_{a e}$ is the aeroelastic moment per unit of span length, $K=(B \omega) / U$ is the reduced frequency, $B$ the deck width and $\omega$ the circular frequency of oscillation, $h$ is the heave displacement and $\dot{h}$ is its time derivative, $\alpha$ is the torsional rotation and $\dot{\alpha}$ its time derivative, $H_{i}^{*}$ and $A_{i}^{*}(i=1, \ldots, 4)$ are the flutter derivatives.

Assuming harmonic forced oscillations $h=h_{0} e^{i \omega t}$ and $\alpha=\alpha_{0} e^{i \omega t}$, where $h_{0}$ and $\alpha_{0}$ are the amplitudes of the oscillations, and also that motion-induced forces are linear functions of the movement, the following expressions are obtained for the identification of the flutter derivatives:

$$
\begin{aligned}
& H_{1}^{*}=-\left(\frac{U}{B f}\right)^{2} \frac{C_{l} \operatorname{Sin} \varphi_{L-h}}{(2 \pi)^{2} h_{0} / B} ; \quad H_{2}^{*}=-\left(\frac{U}{B f}\right)^{2} \frac{C_{l} \operatorname{Sin} \varphi_{L-a}}{(2 \pi)^{2} a_{0}} ; \quad H_{3}^{*}=\left(\frac{U}{B f}\right)^{2} \frac{C_{l} \cos \varphi_{L-a}}{(2 \pi)^{2} a_{0}} ; \\
& H_{4}^{*}=\left(\frac{U}{B f}\right)^{2} \frac{C_{l} \cos \varphi_{L-h}}{(2 \pi)^{2} h_{0} / B} ; \quad A_{1}^{*}=-\left(\frac{U}{B f}\right)^{2} \frac{C_{m} \operatorname{Sin} \varphi_{M-h}}{(2 \pi)^{2} h_{0} / B} ; \quad A_{2}^{*}=-\left(\frac{U}{B f}\right)^{2} \frac{C_{m} \sin \varphi_{M-a}}{(2 \pi)^{2} a_{0}} ; \\
& A_{3}^{*}=\left(\frac{U}{B f}\right)^{2} \frac{C_{m} \cos \varphi_{M-a}}{(2 \pi)^{2} a_{0}} ; \quad A_{4}^{*}=\left(\frac{U}{B f}\right)^{2} \frac{C_{m} \cos \varphi_{M-h}}{(2 \pi)^{2} h_{0} / B} .
\end{aligned}
$$

In eqns. (8), $\varphi_{L-h}, \varphi_{L-a}, \varphi_{M-h}$ and $\varphi_{M-a}$ are the phase lags of the fluctuating aeroelastic lift and moment with respect to the heave and pitch harmonic oscillations. $C_{l}$ and $C_{m}$ are the amplitudes of the non-dimensional aeroelastic lift and moment.

3.3 Identification of vortex induced vibrations from forced oscillations in pitch and heave degrees of freedom

Vortex induced vibrations (VIV) and torsional flutter can be also studied by means of harmonic forced oscillations. In this section a brief summary of the basic formulation is presented, bearing in mind that more detailed derivations can be found in [10] and [11]. Here we refer to the heave degree of freedom, nevertheless similar formulation can be obtained for the pitch degree of freedom.

The body is forced to oscillate in heave according to:

$$
h(t)=h_{0} \sin \left(\omega_{m} t\right)
$$

where $h(t)$, positive upwards, represents the forced oscillation, $h_{0}$ is the displacement amplitude, $\omega_{m}$ is the forced vibration circular frequency and $t$ is time. 
The main components of the unsteady wind force acting on the oscillating bluff-body are the vortex-shedding frequency component and the forced frequency component.

The forced oscillation frequency content of the unsteady lift force is:

$$
L_{m}(t)=L_{0} \sin \left(\omega_{m} t+\beta\right),
$$

being $L_{0}$ the amplitude of the unsteady lift at the excitation frequency, and $\beta$ is the phase shift with respect to the forced oscillation.

Applying the Fourier decomposition it can be obtained:

$$
\begin{gathered}
{\left[a_{m}, b_{m}\right]=\frac{1}{T} \int_{-T}^{T} L(t)\left[\cos \omega_{m} t, \sin \omega_{m} t\right] d t} \\
L_{0}=\sqrt{a_{m}^{2}+b_{m}^{2}} \\
\beta=\tan ^{-1}\left(-b_{m} / a_{m}\right)
\end{gathered}
$$

And the forced frequency component of the unsteady lift force acting on the bluff-body is:

$$
L_{m}(t)=L_{0} \cos \beta \sin \left(\omega_{m} t\right)+L_{0} \sin \beta \cos \left(\omega_{m} t\right) .
$$

In eqn. (14), $L_{m R}=L_{0} \cos \beta$ is the in-phase component, and $L_{m I}=L_{0} \sin \beta$ is the out-ofphase component. The out-of-phase component plays the role of the aerodynamic damping, and the in-phase component plays the role of the aerodynamic stiffness. Positive out-of-phase component indicates that self-excited oscillation may take place.

The non-dimensional in-phase and out-of-phase components of the forced frequency component of the unsteady lift force can be written as:

$$
C_{L R}=\frac{L_{m R}}{\frac{1}{2} \rho U^{2} B}, C_{L I}=\frac{L_{m I}}{\frac{1}{2} \rho U^{2} B} .
$$

\section{DESCRIPTION OF “FLUSINI” SOFTWARE}

\subsection{General description}

FLUSINI acts as intermediary software for setting up in OpenFOAM bluff body aerodynamics problems of interest in bridge engineering. FLUSINI offers to the user a graphical interface for the input of the physical and mesh generation related data of the problem as well as some basic output parameters. Then, the software automatically generates the pertinent folders and files for the complete definition of the OpenFOAM case, for versions 2.1.1 and 2.3.0.

FLUSINI does not consider all the available possibilities for modeling bluff bodies aerodynamics problems. On the contrary, this piece of software deals with some specific kind of problems and geometries. In the same manner, the available modeling approaches and numerical settings are restricted to the ones which have shown good performance based on the authors' previous experience in the field [8, 12]. The main characteristics of the modeling approach for which FLUSINI has been tailored are described in the next paragraphs. 
One of the fundamental goals developing this in-house piece of software is to accurately define 2D or 3D finite volume meshes for FSI problems. The grids, which are parametrically generated, are characterized by the following points:

- The flow domain is rectangular. There are several alternatives such as parabolic or O-ring type meshes; however rectangular domains have been widely used with good results in different problems.

- Parametric block structured regular meshes are employed, since unstructured meshes often require higher computer times in CFD problems according to [13]. Besides, the discretization of flow fluxes in unstructured meshes is more diffusive and a finer spatial discretization is required to obtain grid-independent results [14].

- The mesh topology, that is, the discretization in blocks of the fluid domain, is established by the code for each considered geometry type, without intervention of the user. In Figs. 1a) and $1 \mathrm{~b}$ ) the block topology layout for both the rectangular cylinder and the closed box cross-section are presented. In those figures, a number is assigned to each block. It can be appreciated the higher complexity in the blocks distribution for the closed box deck case.

- No wall functions are adopted for modelling the boundary layer around the bluff body. This entails producing a highly resolved grid attached to the body in order to satisfy the $y^{+}$ requirements of this low Reynolds wall modelling approach. The $y^{+}$is the non-dimensional height of the first layer of cells attached to the body and it is defined as $y^{+}=\left(\delta_{1} u_{*}\right) / v$, where $\delta_{l}$ is the height of the first prismatic grid layer around the deck, $u_{*}$ is the friction velocity and $v$ is the kinematic viscosity. According to Sarkic and co-workers [15], when the values of $y^{+}$are between 2 and 8 , the first layer of cells attached to the wall lies inside the laminar sublayer, and therefore no wall treatment is necessary since the turbulent model equations can be integrated along the viscous sublayer.

- The corners of the bluff bodies are modelled as "sharp".

Currently, in FLUSINI, the URANS (Unsteady Reynolds Averaged Navier-Stokes) approach adopting Menter's $k$ - $\omega$ SST turbulence model has been implemented based on its good accuracy with moderate computational cost. Nevertheless, the software can be readily extended to include Large Eddy Simulation (LES) turbulence modeling capabilities without further difficulties from the programming point of view.

The types of physical simulations considered in FLUSINI software are the following:

- Static bluff body simulations at different angles of attack with the aim of computing force coefficients.

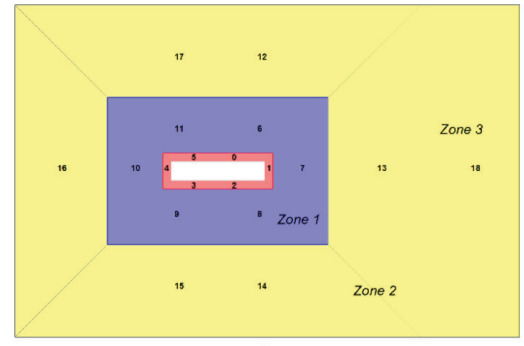

a)

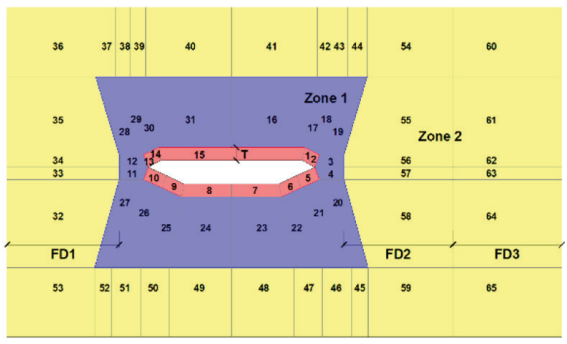

b)

Figure 1: Block topology a) rectangular cylinder geometries, b) streamlined box decks. 
- Forced oscillation in heave, which allows computing heave related flutter derivatives [16] as well as VIV response from the out-of-phase component of the lift coefficient [10]

- Forced oscillation in pitch, for obtaining pitch-related flutter derivatives and also identifying torsional vortex-induced oscillations and torsional flutter prone reduced velocity regions based on the sign of the out-of-phase component of the moment coefficient [17].

In Fig. 2, a flow chart with the basic scheme of the input data to be supplied to FLUSINI and the output files and folders produced by the software is presented. The user must introduce the data related with the definition of the body geometry and the topology of the mesh. Also the data needed for the complete definition of the grid must be given, along with the basic values required for the setup of the initial and boundary conditions of the problem, the choice of simulation type, and some data for setting up the OpenFOAM output. Then, FLUSINI produces the required text files and organizes them in the folders required by OpenFOAM. In the following sections, a detailed description of the operations performed by the piece of software will be provided.

\subsection{Geometry data input and mesh definition with FLUSINI}

In this section the data required by FLUSINI for defining the geometry of the problem along with the process followed by this piece of software to produce the finite volume mesh is explained. FLUSINI creates the blockMeshDict text file from which the blockMesh utility, supplied with the OpenFOAM distribution, generates the mesh.

A three-dimensional, right-handed, Cartesian system $(\mathrm{X}, \mathrm{Y}, \mathrm{Z})$ is considered. The $\mathrm{X}$ axis follows the horizontal direction and it is positive in the stream wise direction; the $\mathrm{Y}$ axis is vertical, positive upwards; and $\mathrm{Z}$ follows the span wise direction, thus it is orthogonal to the flow. In Fig. 3 the subsets of information required for the input of the geometrical data and the definition of the grid are summarized.

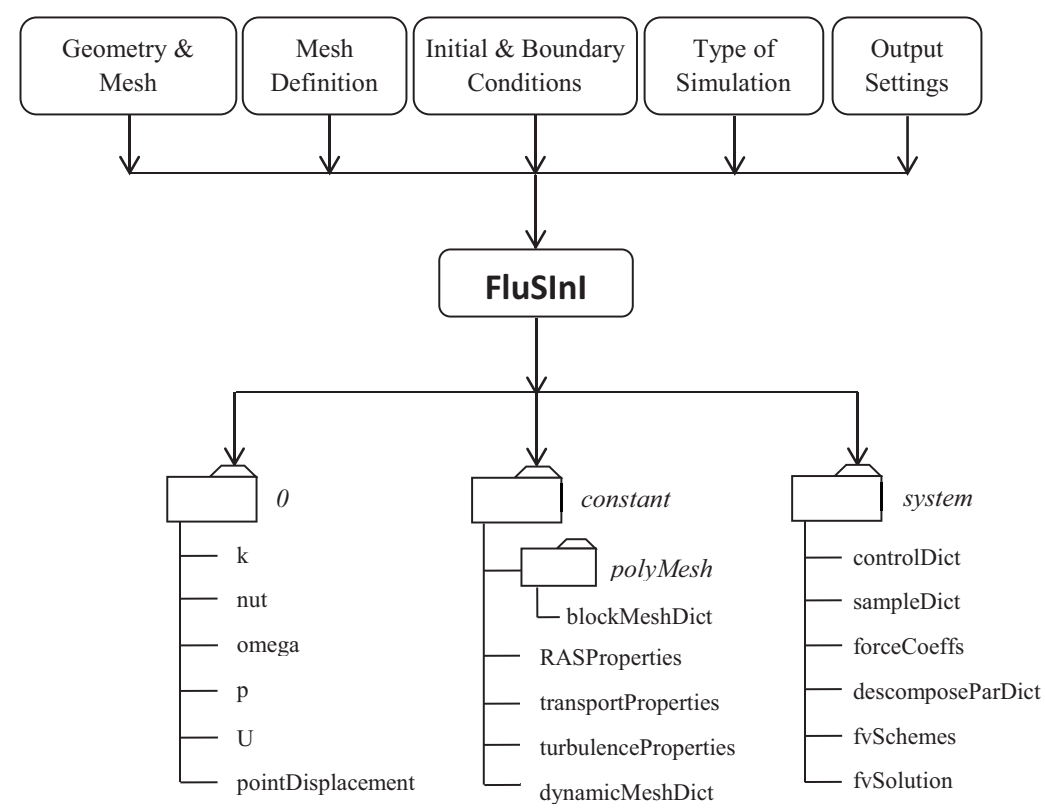

Figure 2: FLUSINI general setup flow chart. 
For setting the problem geometry and the flow domain discretization in blocks the following data must be supplied to FLUSINI: body geometry, overall dimensions of the rectangular flow domain, position of the body inside the flow domain and information related with the location of the blocks' vertices in order to define the topology of the block structured mesh. In Figs. 4 and 5 the forms in FLUSINI for the input of the required geometry and meshing data are presented. Besides, in Table 1 the precise definition of the required data for the
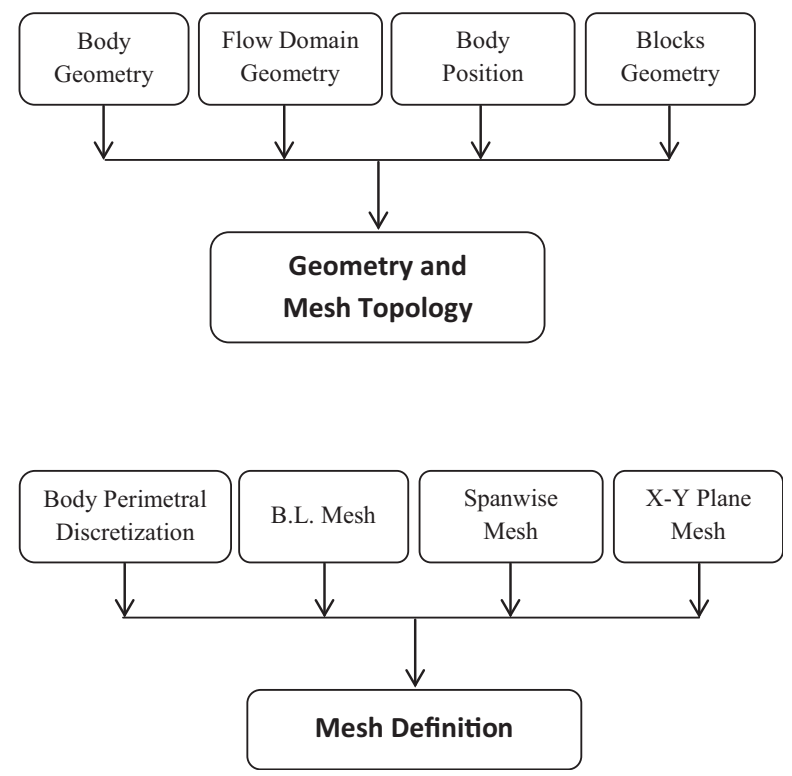

Figure 3: Data subsets for problem geometry and finite volume mesh definition in FLUSINI.

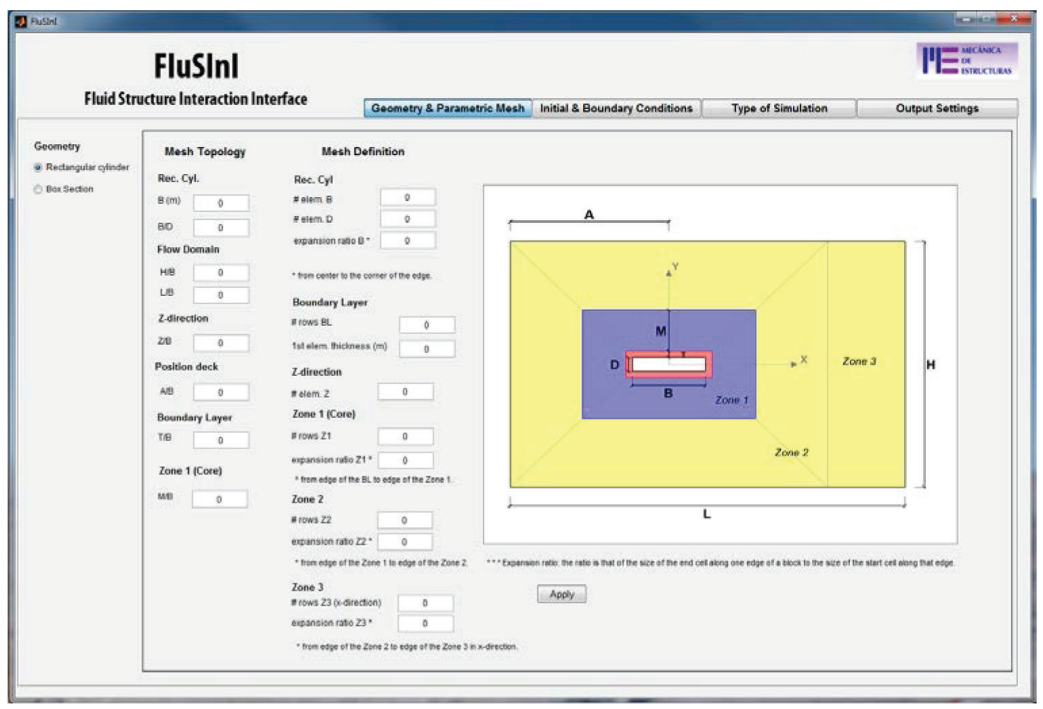

Figure 4: Rectangular cylinder case: FLUSINI's form for geometry and mesh definition. 


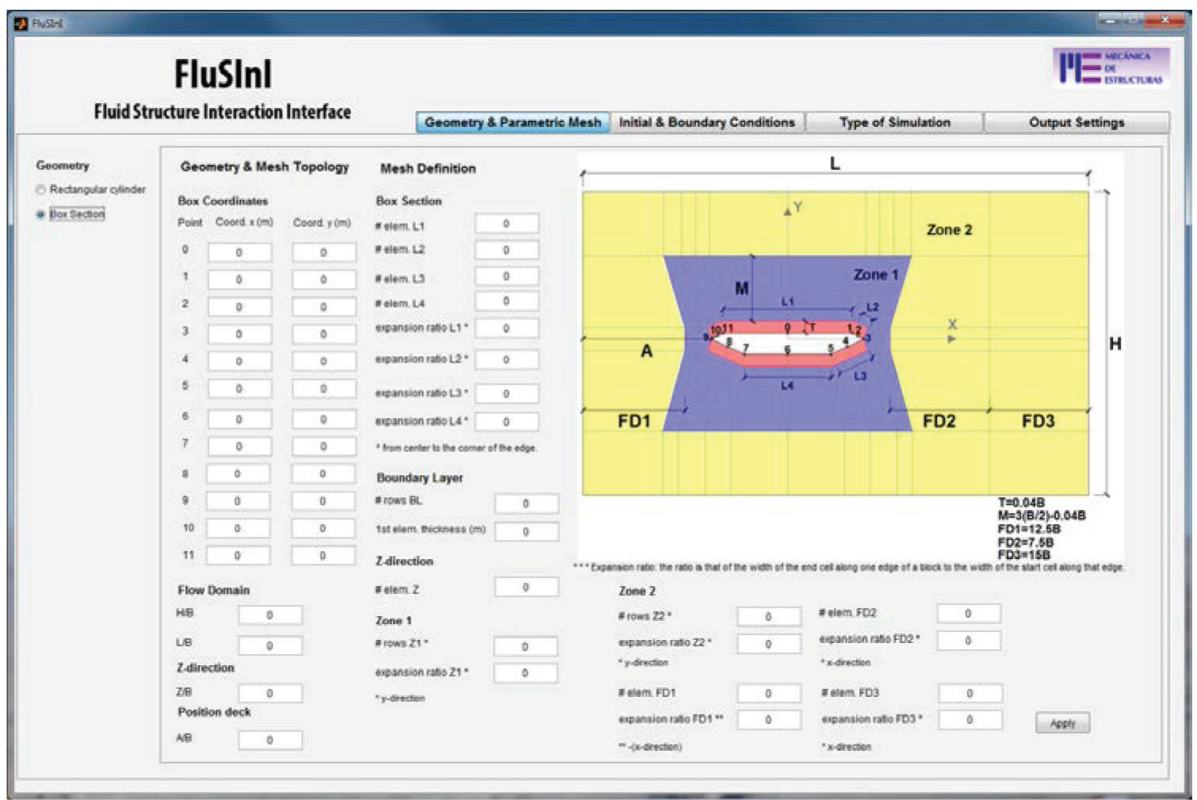

Figure 5: Box girder case: FLUSINI's form for geometry and mesh definition.

geometry and mesh topology setup for each type of problem is presented. In the same manner, in Table 2 the data required for defining the mesh discretization are also explained.

In Fig. 6, an example of a 3D mesh for a rectangular cylinder of width to depth ratio 4:1 is provided. It can be appreciated the ability of the GUI to control the mesh density in both the span wise direction and the XY plane.

\subsection{Initial and boundary conditions}

The input of the initial and boundary conditions of the problem is done using a dedicated form in the software. In first place, the angle of attack of the wind flow related to the bluff body is defined. Also, the velocity of the undisturbed uniform flow and the relative pressure at the outlet are introduced by the user. The turbulence characteristics of the flow at the inlet are defined based on the turbulence intensity and length scale magnitudes. The turbulent kinetic energy per unit mass $k$ is evaluated using the following expression:

$$
k=\frac{3}{2}\left(U T^{\prime}\right) .
$$

Where $U$ is the mean velocity of the undisturbed flow and $T^{\prime}$ is the free stream turbulence intensity.

The specific dissipation rate $\omega$, at the inlet, is defined as:

$$
\omega=\frac{k^{0.5}}{l \beta^{0.25}}
$$

Where 1 is the turbulence length scale and $\beta=0.09$ is one of the closure coefficients of the $k-\omega$ model. 
Table 1: Required data for geometry and mesh topology definition of rectangular cylinder and box girder cases in FLUSINI (See Figs. 4 and 5 for the graphical definition of the geometrical parameters).

\begin{tabular}{|c|c|c|}
\hline Concept & Rectangular cylinder & Box girder \\
\hline $\begin{array}{l}\text { Body } \\
\text { geometry }\end{array}$ & $\begin{array}{l}\text { Width dimension: } B \text { Width to depth } \\
\text { ratio: } B / D\end{array}$ & $\begin{array}{l}\text { Section points coordinates ( } 12 \\
\text { points) }\end{array}$ \\
\hline \multirow[t]{3}{*}{$\begin{array}{l}\text { Flow domain } \\
\text { geometry }\end{array}$} & $\begin{array}{l}\text { Flow domain height to rectangular } \\
\text { cylinder width ratio: } H / B\end{array}$ & \\
\hline & $\begin{array}{l}\text { Flow domain width to rectangular } \\
\text { cylinder width ratio: } L / B\end{array}$ & $\begin{array}{l}\text { As in the rectangular cylinder } \\
\text { case }\end{array}$ \\
\hline & $\begin{array}{l}\text { Span wise dimension to rectangular } \\
\text { cylinder width ratio: } Z / B\end{array}$ & \\
\hline Body position & $\begin{array}{l}\text { Inlet to rectangular cylinder centroid } \\
\text { distance divided by the rectangular } \\
\text { cylinder width: } A / B\end{array}$ & $\begin{array}{l}\text { Inlet to girder's windward } \\
\text { corner distance divided by the } \\
\text { girder width: } A / B\end{array}$ \\
\hline $\begin{array}{l}\text { Blocks } \\
\text { topology }\end{array}$ & $\begin{array}{l}\text { Boundary layer mesh thickness to } \\
\text { rectangular cylinder width ratio: } T / B \\
\text { Zone } 1 \text { thickness to rectangular cylin- } \\
\text { der width ratio: } M / B\end{array}$ & $\begin{array}{l}\text { Fixed parameters: } T=0.04 B \\
M=1.5 B-T F D 1=12.5 B \\
F D 2=75 B ; F D 3=15 B\end{array}$ \\
\hline
\end{tabular}

Impulsive initial conditions are imposed, setting up uniform values at the fluid domain for the velocity, pressure, turbulent kinetic energy, the specific dissipation rate and the kinematic eddy viscosity, based on the values prescribed at the inlet and outlet.

At the surfaces of the rectangular cylinder or streamlined box deck, the no-penetration and no-slip boundary conditions are imposed, while null values for the turbulent kinetic energy

Table 2: Required data for the mesh definition of rectangular cylinder and box girder cases in FLUSINI (See Figs. 4 and 5 for the definition of the graphical geometrical parameters).

\begin{tabular}{|c|c|c|}
\hline Concept & Rectangular cylinder & Box girder \\
\hline $\begin{array}{l}\text { Body perimeter discretiza- } \\
\text { tion }\end{array}$ & $\begin{array}{l}\text { Number of elements and } \\
\text { expansion ratio along } B \\
\text { Number of elements along } D\end{array}$ & $\begin{array}{l}\text { Number of elements and } \\
\text { expansion ratio for } L 1, L 2 \text {, } \\
L 3 \text { and } L 4\end{array}$ \\
\hline $\begin{array}{l}\text { Boundary layer (B.L.) mesh } \\
\text { definition }\end{array}$ & $\begin{array}{l}\text { First element thickness } \\
\text { Number of rows }\end{array}$ & $\begin{array}{l}\text { As in the rectangular cylin- } \\
\text { der case }\end{array}$ \\
\hline $\begin{array}{l}\text { Span wise direction discreti- } \\
\text { zation }\end{array}$ & Number of elements & $\begin{array}{l}\text { As in the rectangular cylin- } \\
\text { der case }\end{array}$ \\
\hline Plane X-Y grid definition & $\begin{array}{l}\text { Zone 1: number of rows and } \\
\text { expansion ratio } \\
\text { Zone } 2 \text {. number of rows and } \\
\text { expansion ratio } \\
\text { Zone 3: number of rows and } \\
\text { expansion ratio }\end{array}$ & $\begin{array}{l}\text { Zone 1: number of rows and } \\
\text { expansion ratio } \\
\text { Zone } 2 \text { : number of rows and } \\
\text { expansion ratio for } \\
F D 1, F D 2 \text { and FD3 }\end{array}$ \\
\hline
\end{tabular}




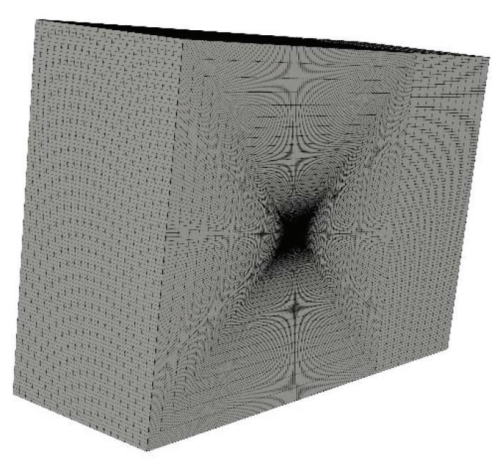

a)

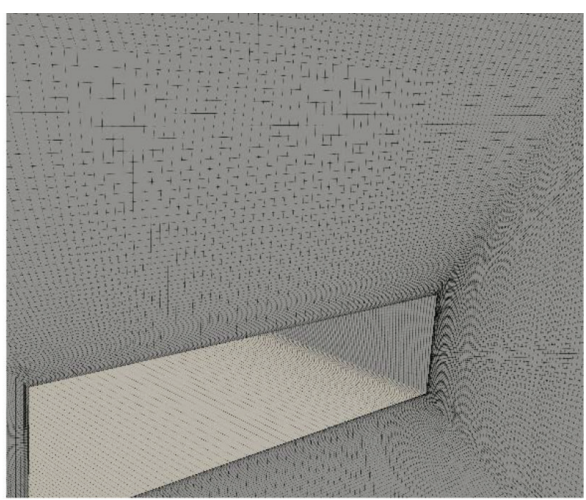

b)

Figure 6: 3D grid for a 4:1 rectangular cylinder a) General view, b) detail close to the body.

per unit mass are prescribed, and the specific dissipation rate at the walls is obtained using the following expression:

$$
\omega_{\mathrm{wall}}=\frac{60 v}{0.075\left(\delta_{1}\right)^{2}},
$$

where $v$ and $\delta_{1}$ were defined in the expression of $y^{+}$introduced in section 4.1. FLUSINI creates for each type of simulation the required text files $k$, nut, omega, $p$ and $U$, located inside the " 0 " (zero) folder of the OpenFOAM case.

Furthermore, with the data provided by the user, FLUSINI generates the forceCoeffs dictionary, allocated in the "system" directory, comprising the required information for computing the forces and force coefficients acting on the body, and generating the corresponding output files during the simulation.

\subsection{Types of simulations}

The user must select the type of simulation which is going to be computed by means of the OpenFOAM solver. Accordingly, the required input data must be supplied, and then FLUSINI creates the pertinent dictionaries for the OpenFOAM case. The available types of simulation are the following:

- Static: the body remains fixed along the time marching simulation. In this way the force coefficients at different angles of attack (see the initial and boundary condition section) can be computed from the lift, drag and moment time histories.

- Heave oscillation: the body surpasses forced oscillations in heave following a sinusoidal function. The user must provide the amplitude of oscillation as well as the frequency of the excitation in Hz. The output allows studying the vortex induced vibration response of the body and the extraction of the heave related flutter derivatives at different reduced velocities.

- Pitch oscillation: in this case the body is harmonically excited with frequency and rotation amplitude provided by the user. The purpose of this type of simulation is computing the pitch related flutter derivatives and the identification of torsional vortex induced vibration or torsional flutter prone regions, as a function of the reduced velocity. 


\subsection{Output settings}

In a specific form in the GUI the output settings must be supplied. Two transient solvers for handling the pressure-velocity coupling of the Navier-Stokes equations are considered: pimpleFoam, for the computation of the force coefficients (static body), and pimpleDyMFoam, for the forced oscillation simulations (moving body and dynamic mesh). According to Holzmann [18], the PIMPLE algorithm is a blend of the PISO and SIMPLE algorithms, reaching a steady-state solution for each time step, using under-relaxation, aiming at providing stability to the iterative process. Once the solution is obtained, the solution process advances to the next time step. This algorithm allows adopting Courant numbers higher than one. The pimpleDyMFoam solver is the moving mesh version of the PIMPLE algorithm previously described, adopting the ALE formulation. These solvers correspond to the standard formulation in the OpenFOAM distribution, and have not been modified by the authors.

The coupling between the mesh movement and the resolution of the flow is achieved applying the Conventional Serial Staggered approach, that is profusely used in fluid-structure interaction applications, and has provided accurate results for the application cases in section 5 .

The user must provide the following data: initial time step size, time length of the simulation and the maximum Courant Number. Besides this, the writing interval for the output files and the number of the most recent time steps for which automatically save the output files along the simulation must be supplied. Another important parameter is the number of processors considered for parallel computation of the simulation.

With these data, FLUSINI generates the following files, which are located inside the system folder: controlDict, decomposeParDict, fvSchemes and fvSolution. It must be noted that

Table 3: Default linear-solvers in the fvSolution dictionary.

\begin{tabular}{ll}
\hline Variable & Solver \\
\hline p/pFinal & GAMG \\
U/Ufinal & PBiCG \\
k/kFinal/m/mFinal & SmoothSolver \\
cellDisplacement & GAMG \\
\hline
\end{tabular}

Table 4: Default numerical schemes in the fvSchemes dictionary.

\begin{tabular}{ll}
\hline Category & Numerical schemes \\
\hline ddtSchemes & default Euler \\
gradSchemes & default cellLimited Gauss linear 0.3 \\
& div(phi,U) Gauss linearUpwindV grad(U) \\
& div(phi,k) Gauss upwind div(phi,omega) Gauss \\
divSchemes & upwind \\
& div $(($ nuEff*dev $(\mathrm{T}(\operatorname{grad}(\mathrm{U})))))$ Gauss linear \\
laplacianSchemes & default Gauss linear limited 0.8 \\
interpolationSchemes & default linear \\
snGradSchemes & default corrected limited 0.8 \\
\hline
\end{tabular}


the numerical settings, which cannot be accessed through the GUI, are defined a priori based on previous successful experiences by the authors, although they can be modified by the user in the corresponding text files. In Tables 3 and 4, the default values for the fvSchemes and fvSolution dictionaries are provided.

\section{APPLICATION EXAMPLES}

In the following sections, the results obtained for static and forced oscillation simulations of both, a rectangular cylinder and a streamlined closed box deck cross section, are provided.

The goal of this section is to show the performance of FLUSINI as a GUI for OpenFOAM, setting up the simulations in a rigorous fashion, making straightforward obtaining accurate numerical results for the aerodynamic and aeroelastic phenomena of interest. Hence, studies of sensitivity of grid and time step resolution are not provided since the mesh characteristics are similar to ones described in previous works by the authors $[8,12]$, where verification studies are provided. In the same manner, complete sets of reduced velocities or angles of attack were not computed, since the target has not been the detailed numerical study of a particular problem, but the assessment of the feasibility of the software for the intended applications.

In the choice of the application examples, the existence in the literature of both, wind tunnel data and CFD-based simulations, has been a key issue. The availability of experimental data for the proper validation of the numerical results reported herein is of utmost importance; furthermore, the comparison of our CFD work with similar numerical simulations authored by other researchers would allow gaining confidence in FLUSINI and the techniques and numerical settings adopted in this bespoke piece of software.

\subsection{Ratio 2:1 rectangular cylinder}

This small B/D ratio rectangular cylinder shows a separated flow from the leading edge, which does not reattach on the upper and lower surfaces of the cylinder. It exhibits several types of aerodynamic instabilities and has been extensively studied by means of wind tunnel tests and also computer simulations.

\subsubsection{Grid definition}

The data required by FLUSINI for the definition of the geometry and the structured mesh were introduced in the form shown in Fig. 4. For this application example, in Table 5 the values of the parameters previously defined in Tables 1 and 2 are provided.

Based on the data supplied by the user (Table 5) and the geometrical relations implemented in the software, the finite volume mesh can be defined. In Fig. 7 some images of this grid are presented. The images make clear the ability of FLUSINI to produce high density block structured rectangular meshes suitable for low Reynolds wall modeling type simulations in aerodynamics problems of rectangular cylinders.

The total number of cells in the mesh is 151640 . For this rectangular cylinder, the range of Reynolds numbers, $\mathrm{Re}_{B}$, in the simulations that are going to be presented next is $\left(3.4 \times 10^{4}\right.$, $\left.6.4 \times 10^{4}\right)$. For the maximum $\operatorname{Re}_{B}$ the mean value of the first cell non-dimensional height $y^{+}$ is about 1.15 , while the maximum value of $y^{+}$is close to 6.6.

\subsubsection{Aerodynamic response of the fixed 2:1 rectangular cylinder}

With the purpose of validating the finite volume mesh previously described, as well as the $2 \mathrm{D}$ URANS approach and the $k$ - $\omega$ SST turbulence model, the force coefficients of the fixed 
Table 5: Required data for the mesh definition for rectangular cylinder case in FLUSINI (See Fig. 4 for the definition of the graphical geometrical parameters).

\begin{tabular}{|c|c|}
\hline Concept & 2:1 Rectangular Cylinder \\
\hline Body geometry & $B=0.08 m ; B / D=2$ \\
\hline Flow domain geometry & $\begin{array}{l}H / B=30 ; L / B=40 \\
Z / B=1\end{array}$ \\
\hline Body position & $A / B=15$ \\
\hline Blocks topology & $T / B=0.009 ; M / B=0.70$ \\
\hline Body perimeter discretization & $\begin{array}{l}\text { No. cells } B=160 \\
\text { Exp. ratio }=0.5 \\
\text { No. cells } D=100 \\
\text { Exp. ratio }=0.5\end{array}$ \\
\hline Boundary layer mesh definition & $\begin{array}{l}\text { No. rows bound. layer }=12 \\
1^{\text {st }} \text { cell thickness }=4.15 e-5 \mathrm{~m}\end{array}$ \\
\hline Span wise direction discretization & No. cells $Z=1$ \\
\hline Plane $\mathrm{X}$-Y grid definition & $\begin{array}{l}\text { No. rows } Z 1=100 \\
\text { Exp. ratio }=10 \\
\text { No. rows } Z 2=170 \\
\text { Exp. ratio }=10 \\
\text { No. rows } Z 3=50 \\
\text { Exp. ratio }=1\end{array}$ \\
\hline
\end{tabular}

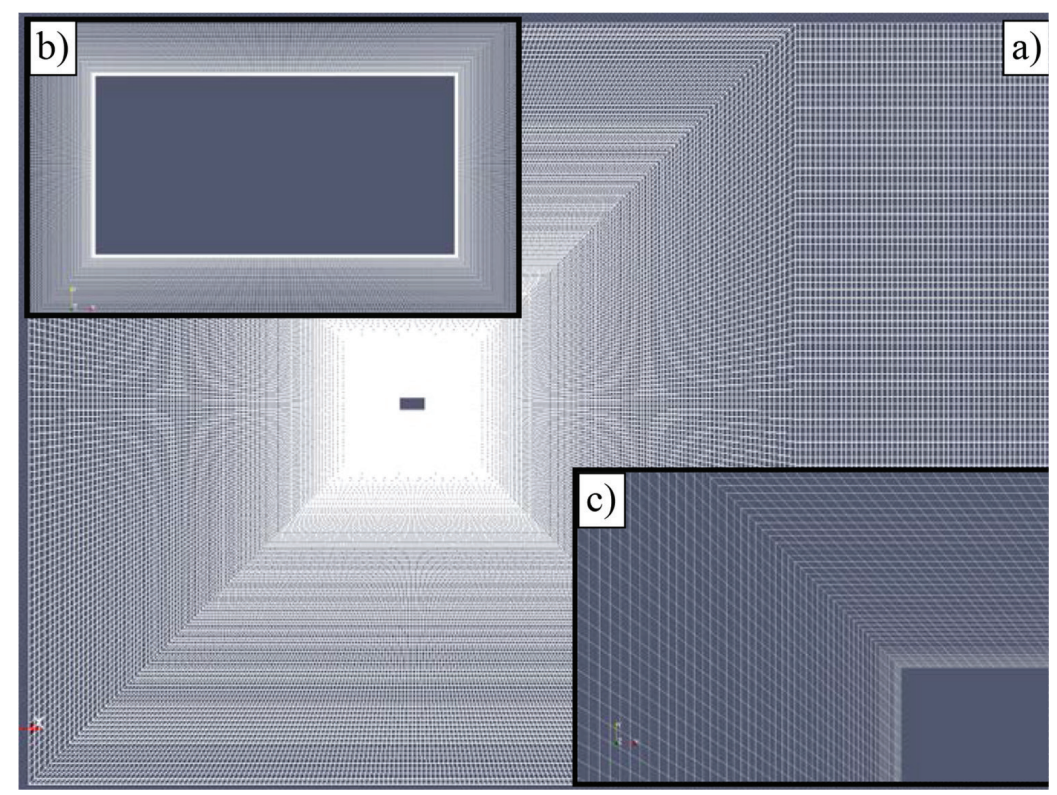

Figure 7: $B / D=2$ rectangular cylinder mesh. a) General view, b) Mesh around the body; c) Detail of the mesh at the corners. 
Table 6: $B / D=2$ rectangular cylinder: results and validation. The standard deviation of the force coefficients is identified with the prime symbol.

\begin{tabular}{lccc}
\hline & $\boldsymbol{S} \boldsymbol{T}$ & $\boldsymbol{C D}$ & $\boldsymbol{C L}^{\prime}$ \\
\hline Present simulation & 0.081 & 0.73 & 0.74 \\
CFD [21] & 0.084 & 0.72 & 0.23 \\
Experimental $[19,20]$ & 0.089 & 0.78 & 0.55 \\
\hline
\end{tabular}

rectangular cylinder are computed and compared with the wind tunnel data reported in [19] and [20]. In the same manner, the numerical results reported in [21] for the same rectangular cylinder ratio are included in the comparison.

In Table 6 , the numerical results obtained at $\mathrm{Re}_{\mathrm{B}}=4.4 \times 10^{4}$ are provided, presenting good agreement in the Strouhal number and the drag coefficient with the experimental data. On the other hand, the standard deviation of the lift coefficient overestimates the value in the experimental reference. However, according to Brusiani and co-workers [22], 2D simulations show perfect correlation of the flow structures, since the 3D vortical structures cannot be simulated. Hence, an overestimation in the amplitude of the force coefficients must be expected in 2D simulations.

\subsubsection{Heave degree of freedom forced oscillation}

The ratio 2:1 rectangular cylinder exhibits vortex-induced vibrations for reduced velocities close to 3 , but also galloping for reduced velocities higher than 8 . In order to validate the capability of FLUSINI for the setup of this kind of simulations, the peak of positive out-ofphase component of the lift coefficient, which identifies the reduced velocity region prone to vortex induced vibrations, is identified. The amplitude of the oscillations is $h_{0} / D=0.1$, as in [11].

In Fig. 8, the out-of-phase component of the lift coefficient and the phase angle between the lift force and the forced oscillation are reported. The region of reduced velocities prone to vortex induced vibration has been correctly identified and the numerical values obtained are close to the 2D URANS $k-\varepsilon$ simulations in [11], as well as the experimental references.

\subsubsection{Pitch degree of freedom forced oscillation}

The $B / D=2$ rectangular cylinder exhibits also torsional vortex induced vibrations for reduced velocities in the vicinity of 3 . In the same manner, torsional flutter can take place at higher

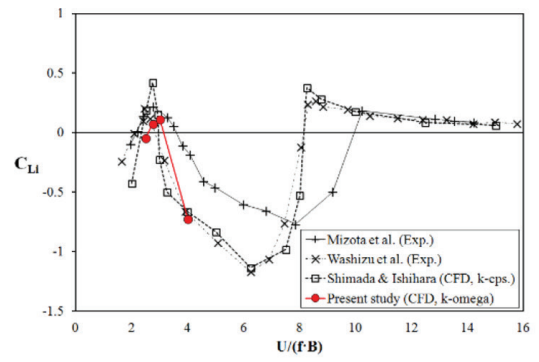

a)

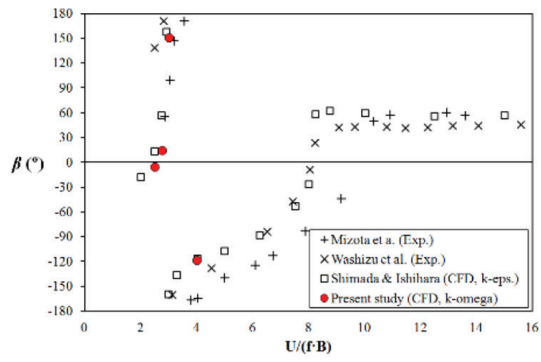

b)

Figure 8: $B / D=2$ rectangular cylinder: a) out-of-phase component of the unsteady lift coefficient and $b$ ) phase angle. 


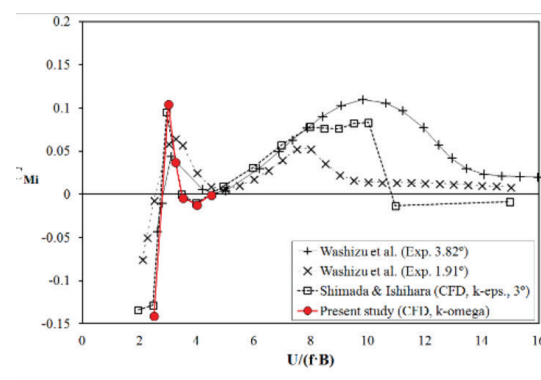

a)

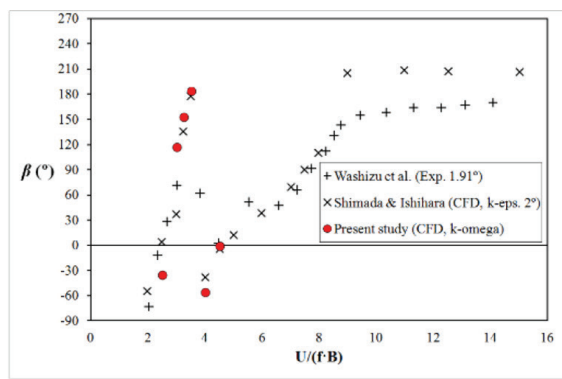

b)

Figure 9: $B / D=2$ rectangular cylinder: a) out-of-phase component of the unsteady moment coefficient and b) phase angle.

values. Using FLUSINI, the torsional vortex induced vibration region has been correctly identified imposing an amplitude of oscillation of $3^{\circ}$.

Also in this case, the numerical results are close to the numerical simulations in [11] and the experimental results in [17] employed for validation (see Fig. 9).

\subsection{Streamlined box cross section}

Streamlined closed box cross sections are common choice nowadays for the decks of long span bridges. They have been extensively studied by means of wind tunnel experiments, and they have been a frequent study case for CFD applications.

For this application case, the geometry of the streamlined box deck in [15] has been chosen, since in the aforementioned reference a complete set of high quality experimental and numerical results (using OpenFOAM as solver) are provided, which allow a complete validation of the numerical simulations reported herein. In Fig. 10 the geometry of the deck and the fundamental dimensions can be appreciated.

\subsubsection{Grid definition}

In Table 7 the data required by FLUSINI for the generation of the 2D structured mesh are presented. The parameters are given following the same order as in Tables 1 and 2 .

In Fig. 11, three images of the structured mesh generated using FLUSINI are provided. The total number of cells is 263810 . The simulations, whose results are reported next, have been conducted at $\operatorname{Re}_{B}=10^{5}$. Regarding the non-dimensional first layer height $y^{+}$, the mean value is about 0.95 , while the maximum value is around 3.7 .

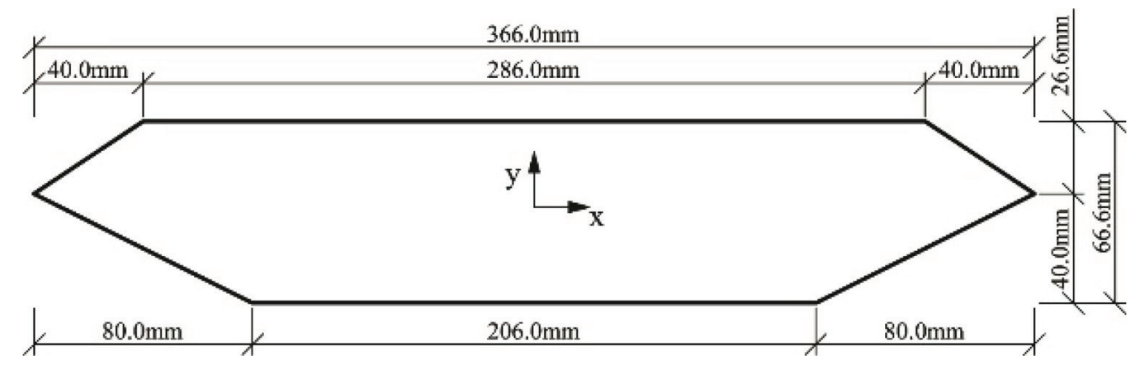

Figure 10: Geometry of the closed box cross section [15]. 
Table 7: Mesh definition parameters for the closed box in FLUSINI.

\begin{tabular}{llll}
\hline Concept & Box girder & \\
\hline & Point & Coord. $X(\mathrm{~m})$ & Coord. $Y(\mathrm{~m})$ \\
0 & 0 & 0.0314 \\
& 1 & 0.143 & 0.0314 \\
& 2 & 0.163 & 0.0181 \\
& 3 & 0.183 & 0.0048 \\
Body & 4 & 0.143 & -0.0152 \\
geometry & 5 & 0.103 & -0.0352 \\
& 6 & 0 & -0.0352 \\
& 7 & -0.103 & -0.0352 \\
& 8 & -0.143 & -0.0152 \\
& 9 & -0.183 & 0.0048 \\
& 10 & -0.163 & 0.0181 \\
& 11 & -0.143 & 0.0314 \\
& $B=0.366$ &
\end{tabular}

Flow domain $\quad H / B=27 ; L / B=37 ; Z / B=1$

geometry

Body position $\quad A / B=13$

Blocks $\quad T=0.04 B=0.001464 m ; M=1.5 B-T=0.53436 m$

topology $F D 1=12.5 B=4.575 m ; F D 2=7.5 B=2.745 m ; F D 3=15 B=5.49 m$

Concept Box girder

Body No. cells $L 1=255$ Exp. ratio $=0.2 ;$ No. cells $L 2=75$ Exp. ratio $=0.5$

perimeter No. cells $L 3=110$ Exp. ratio $=0.25$; No. cells $L 4=145$ Exp. ratio $=0.2$

discretization

B. Layer mesh No. rows boundary layer $=50 ; 1^{\text {st }}$ cell thickness $=7.32 e-5 \mathrm{~m}$ definition

Span wise $\quad$ No. cells $Z=1$

direction

discret.

Plane X-Y grid $\quad$ No. rows $Z 1=70$, Exp. ratio $=30 ;$ No. rows $Z 2=35$, Exp. ratio $=20$

definition No. rows $F D 1=60$, Exp. ratio $=20 ;$ No. rows $F D 2=180$, Exp. ratio $=10$ No. rows $F D 3=50$, Exp. ratio $=5$

5.2.2 Aerodynamic response of the fixed streamlined box cross section

As an initial validation of the numerical simulations carried out, the force coefficients at angles of attack $0^{\circ},-4^{\circ}$ and $-6^{\circ}$ are presented in Fig. 12. The numerical results obtained using the combination of FLUSINI as GUI and OpenFOAM as solver are presented along with the experimental data and the URANS simulations reported in [15]. The general agreement amongst the data is good, particularly between the two CFD simulations. 


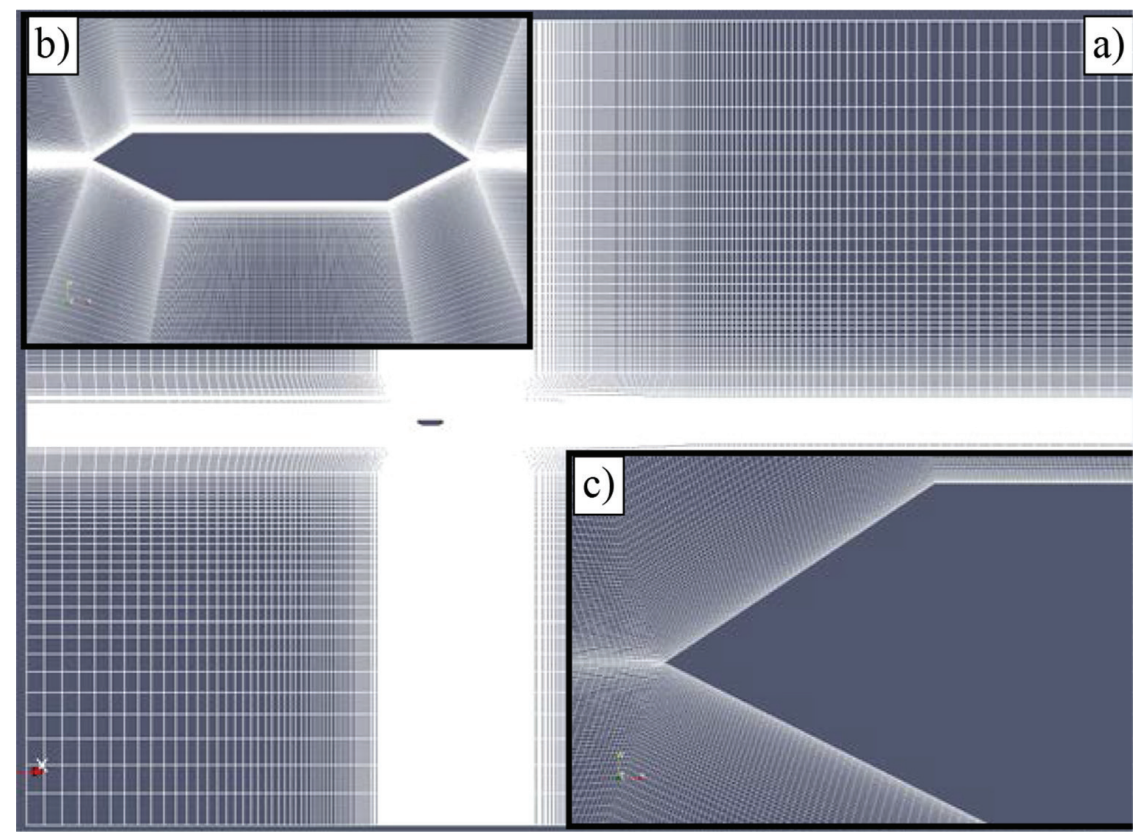

Figure 11: Box deck mesh. a) General view, b) Mesh around the body; c) Detail of the mesh at the corners.

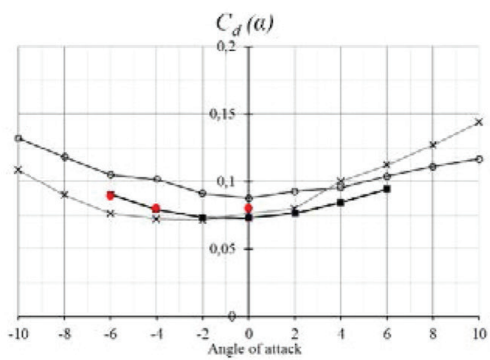

a)

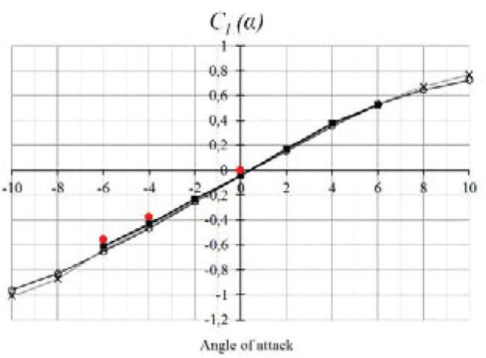

b)

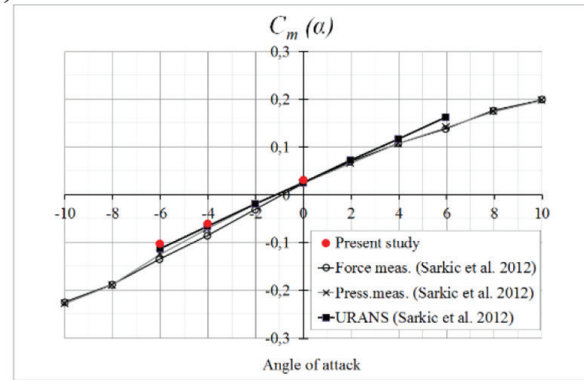

c)

Figure 12: Closed box deck force coefficients: present results and comparison with experimental and numerical data from Sarkic et al. (2012) [15] a) Drag coefficient b) Lift coefficient c) Moment coefficient. 

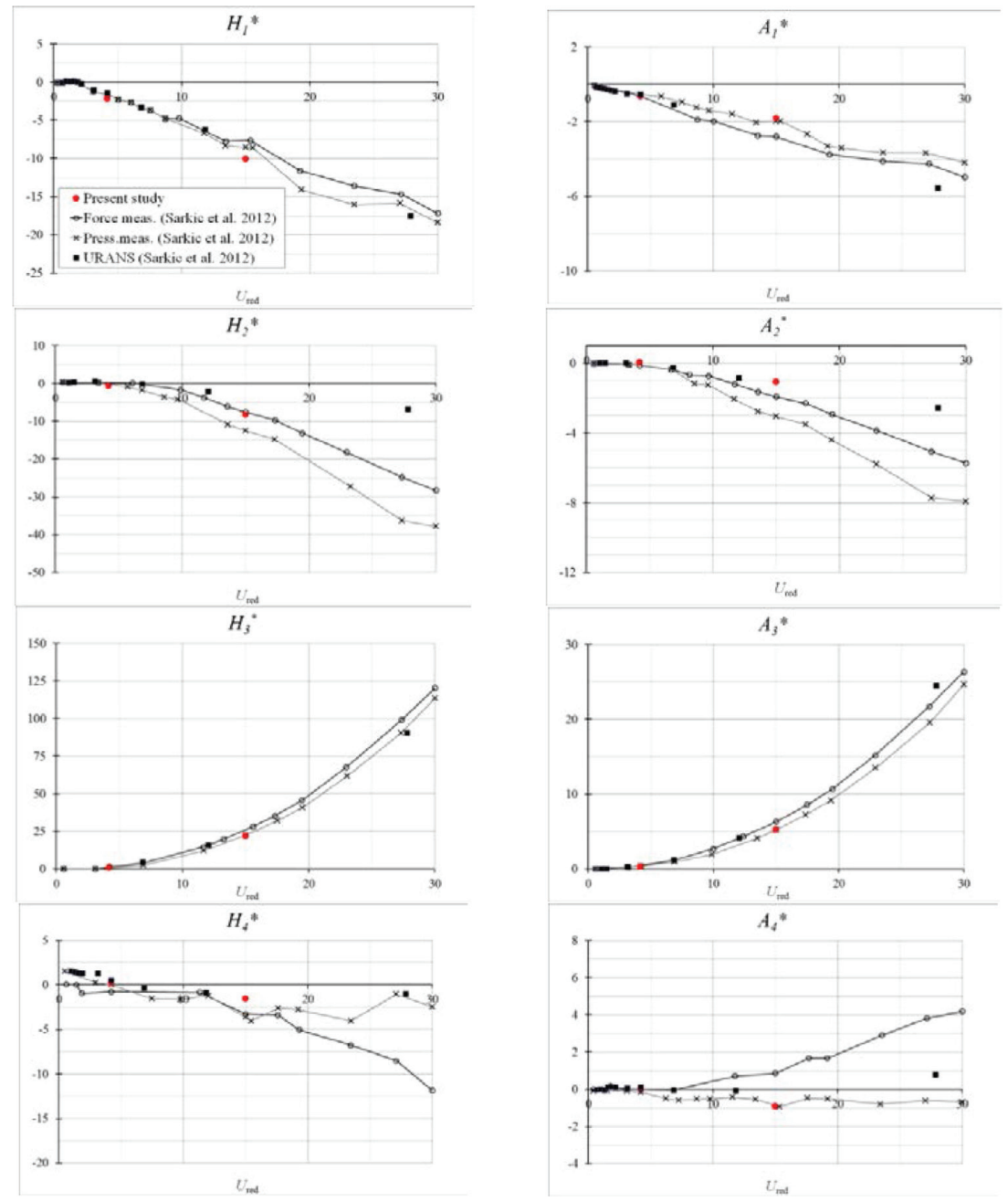

Figure 13: Closed box flutter derivatives: results and comparison with data in [15].

5.2.3 Flutter derivatives of the streamlined box cross section

The set of eight flutter derivatives related with heave and pitch degrees of freedom are computed from forced harmonic oscillations at a $0^{\circ}$ angle of attack. Two reduced velocities $U_{\text {red }}=U j(f \cdot B)=4.2$ and 15 have been considered.

In Fig. 13 the simulations reported in this work show similar results when they are compared with the wind tunnel experiments and numerical simulations in [15].

\section{CONCLUSIONS}

An in-house piece of software named FLUSINI has been presented, which is capable of acting as a GUI for setting up the required data for mesh generation and static and forced oscillation simulations in heave and pitch degrees of freedom using the open source CFD solver OpenFOAM. The programming language has been MATLAB. 
The first application example has been a 2:1 rectangular cylinder. Using FLUSINI, a fully structured grid has been produced, and the data for the setup of a fixed body simulation at $0^{\circ}$ angle of attack have been supplied. Furthermore, forced oscillation simulations in both heave and pitch degrees of freedom have been carried out using the setup created by this piece of software. The numerical results have been in good agreement with available experimental data.

The second application example has been a streamlined closed box deck with the same geometry as in Sarkic et al. (2012) [15]. FLUSINI has successfully setup the numerical models. The results obtained are similar to those reported in the previously mentioned reference.

It has been proved that FLUSINI offers a graphical alternative for the setup of fixed body and forced oscillation simulations in OpenFOAM. The graphical capabilities of this software provide a user-friendly interface for users unfamiliar to OpenFOAM's text files based workflow. More importantly, no deterioration in numerical results has been found when the simulations created with FLUSINI have been compared with standard CFD simulations in the literature.

This piece of software can be integrated into an optimal design solver to produce the required CFD models linked with the modified designs in the frame of shape optimization problems.

The code capabilities can be extended in multiple directions such as: including the setup of LES based simulations, extending the available turbulence models, adding postprocessing capabilities such as the generation of instantaneous and time-averaged pressure coefficient distributions along the section...etc.

\section{ACKNOWLEDGEMENTS}

This work has been funded by the Galician Government (including FEDER funding) with reference GRC2013-056 and by the Spanish Ministry of Economy and Competitiveness (MINECO) with reference BIA2013-41965-P

\section{REFERENCES}

[1] Alvarez, A.J., Nieto, F., Kwok, K.C.S. \& Hernandez, S., Aerodynamic performance of twin-box decks: A parametric study on gap width effects based on validated 2D URANS simulations. Journal of Wind Engineering and Industrial Aerodynamics 2018, 182, pp. 202-221.

[2] Cid Montoya, M., Hernandez, S. \& Nieto, F., Shape optimization of streamlined decks of cable-stayed bridges considering aeroelastic and structural constraints. Journal of Wind Engineering and Industrial Aerodynamics 2018, 177, pp. 429-455.

[3] Barrero, A., Alonso, G., Meseguer, J. \& Astiz, M.A., Ensayos en tunel de viento de un modelo aeroelastico del arco del puente sobre el no Tajo "Arcos de Alconetar". Hormigony Acero 2007, 245, pp. 33-40. (In Spanish).

[4] Patruno, L., Accuracy of numerically evauated flutter derivativesof bridge deck sections using RANS: Effects on the flutter onset velocity. Eng. Structures 2015, 89, pp. 49-65.

[5] Haque, M.N., Katsuchi, H., Yamada, H. \& Nishio, M., Strategy to develop efficient grid system for flow analysis around two-dimensional bluff bodies. KSCE Journal of Civil Engineering 2015, 20(5), pp. 1913-1924.

[6] Wilcox, D.C., Turbulence modeling for CFD 2006. 3rd Edition; DCW Industries, Inc.

[7] Menter, F. \& Esch, T., Elements of industrial heat transfer prediction. Proc. 16th Brazilian Congress of Mechanical Engineering 2001, Invited lectures 20, pp. 117-127. 
[8] Nieto, F., Hargreaves, D.M., Owen, J.S. \& Hernandez, S., On the applicability of 2D URANS and SST $k$ - $\omega$ turbulence model to the fluid-structure interaction of rectangular cylinders. Engineering Applications of Computational Fluid Mechanics 2015, 9(1), pp. 157-173.

[9] Simiu, E. \& Scanlan, R.H., Wind effects on structures 1996. 3rd Edition; John Wiley \& Sons, Inc.

[10] Washizu, K, Ohya, A., Otsuki, Y. \& Fujii, K., Aeroelastic instability of rectangular cylinders in a heaving mode. Journal of Sound and Vibration 1978, 59(2), pp. 195-210.

[11] Shimada, K. \& Ishihara, T. Predictability of unsteady two-dimensional k-e model on the aerodynamic instabilities of some rectangular prisms. Journal of Fluids and Structures 2012, 28, pp. 20-39.

[12] Nieto, F., Owen, J.S., Hargreaves, D.M. \& Hernandez, S., Bridge deck flutter derivatives: Efficient numerical evaluation exploiting their interdependence. Journal of Wind Engineering and Industrial Aerodynamics 2015, 136, pp. 138-150.

[13] Zhang, Y., Jia, Y., Wang, S.S.Y. \& Altinakar, M., Composite structured mesh generation with automatic domain decomposition in complex geometries. Engineering applications of computational fluid mechanics 2013, 7(1), pp. 90-102.

[14] Mannini, C., Soda, A. \& Schewe, G., Numerical investigation on the threedimensional unsteady flow past a 5:1 rectangular cylinder. Journal of Wind Engineering and Industrial Aerodynamics 2011, 99(4), pp. 469-482.

[15] Sarkic, A., Fisch, R., Hoffer, R. \& Bletzinger, K., Bridge flutter derivatives based on computed, validated pressure fields. Journal of Wind Engineering and Industrial Aerodynamics 2012, pp. 104-106, 141-151.

[16] Sarkar, P.P., Caracoglia, L., Haan F.L., Sato, H. \& Murakoshi, J., Comparative and sensitive study of flutter derivatives of selected bridge deck sections, Part 1: Analysis of inter-laboratory experimental data. Engineering Structures 2009, 31, pp. 158-169.

[17] Washizu, K, Ohya, A., Otsuki, Y. \& Fujii, K., Aeroelastic instability of rectangular cylinders in a torsional mode. Journal of Sound and Vibration 1980, 72(4), pp. 507-521.

[18] Holzmann, T. Mathematics, Numerics, Derivations and OpenFOAM 2017, Holzmann CFD, 4th Edition.

[19] Nakaguchi, H., Hashimoto, K. \& Muto, S., An experimental study on aerodynamic drag of rectangular cylinders. Journal of the Japan Society of Aeronautical and Space Sciences 1968, 16, pp. 1-5.

[20] Sakamoto, H., Haniu, H. \& Kobayashi, Y., Fluctuating force acting on rectangular cylinders in uniform flow (on rectangular cylinders with fully separted flow). Transactions of the Japan Society of Mechanical Engineers, Series B 55 1989, 516, pp. 2310-2317.

[21] Shimada, K. \& Ishihara, T., Application of a modified $k-\varepsilon$ model to the prediction of aerodynamic characteristics of rectangular cross-section cylinders. J. Fluids Struct. 2002, 16(4), pp. 465-485.

[22] Brusiani, F., de Miranda, S., Patruno, L., Ubertini, F. \& Vaona, P., On the evaluation of bridge deck flutter derivatives using RANS turbulence models. Journal of Wind Engineering and Industrial Aerodynamics 2013, 119, pp. 39-47. 